\title{
Spectrum Markets for Wireless Services
}

\author{
Junjik Bae*, Eyal Beigman ${ }^{\dagger}$, Randall Berry*, Michael L. Honig*, Hongxia Shen*, Rakesh Vohra ${ }^{\dagger}$, and Hang Zhou* \\ *EECS Department \\ Northwestern University, Evanston, IL 60202 \\ $\{\mathrm{mh}$, rberry $\} @$ eecs.northwestern.edu, hang-zhou@northwestern.edu \\ junjik@u.northwestern.edu, hshen@eecs.northwestern.edu \\ $\dagger$ CMS-EMS, Kellogg School of Management \\ Northwestern University, Evanston, IL 60208 \\ \{r-vohra, e-beigman\}@ northwestern.edu
}

\begin{abstract}
It has been widely recognized that the current under-utilization of spectrum across many bands could be alleviated through the application of spectrum markets. So far, discussions of market mechanisms for spectrum allocations have focused primarily on secondary markets, which are managed by licensees. Here we explore the consequences of lifting current restrictions on allocations and ownership, and allowing more extensive markets for allocating spectrum across locations, times, and diverse sets of applications (e.g., broadcast, cellular, broadband data, emergency, etc).

To motivate our discussion we first estimate the achievable rate per user that could be provided by sharing a large portion of the spectrum suitable for cellular and broadcast types of services. Our results suggest that in general the demand for spectrum may exceed supply implying that market mechanisms are needed to avoid a tragedy of the commons (i.e., associated with an alternative commons model). We then discuss a twotier spectrum market structure for wireless services in which licenses for spectrum assets at particular locations are traded as commodities. Spectrum owners can choose to rent or lease their spectrum assets via spot markets at particular locations. Such an approach may lower barriers to entry into the wireless services market thereby facilitating competition and the introduction of new services.
\end{abstract}

\section{INTRODUCTION}

In the U.S., most commercial spectrum is packaged as licenses that grant the holder exclusive use of the band for ten years. Existing licenses are typically renewed and new licenses are allocated by the Federal Communications Commission (FCC) via auction. ${ }^{1}$ Moreover, the services that can be offered in given bands are decided in a top-down manner by the FCC. $^{2}$ A variety of studies have demonstrated that this policy has resulted in a great deal of spectrum lying idle. ${ }^{3}$ It has been estimated that in some densely populated areas, spectrum occupancy is at most $25 \%$.

This research was supported by the National Science Foundation under grant CNS-0519935.

${ }^{1}$ Auctions have only recently been used for allocating spectrum. Previously, comparative hearings and lotteries were used to allocate licenses. In fact, only a small fraction of the spectrum in use today has been allocated via auctions [34].

${ }^{2}$ Newly auctioned licenses provide more flexibility in this regard, but are still limited in the types of services that can be offered.

${ }^{3}$ See for example the spectrum occupancy measurements reported at www. sharedspectrum. com.
There are several reasons why current spectrum policy has led to this low utilization. The main reason is that spectrum is currently allocated to many applications, which do not make efficient use of the assigned spectrum. In addition, the sporadic and cumbersome nature of the current allocation process for commercial services (e.g., cellular) prompts wireless service providers to acquire large portions of spectrum across different geographic areas in anticipation of future needs. Further inefficiencies then arise due to the coarse temporal and spatial scale over which spectrum is allocated. For example, a potential service provider cannot in general buy a license to use particular spectrum for only a few hours each night.

In 2003, the FCC sought to alleviate these problems by allowing a primary licensee to sub-let spectrum access to other secondary incumbents [17]. ${ }^{4}$ In particular, the FCC made it easier for spectrum to be provided to secondary users for any length of time, time of day, size of coverage area, and frequency or number of channels up to the full capacity of the original license. Nevertheless, the market to sub-let licenses is not an active one. According to [39], a reason for this is that license holders are reluctant to sub-let for fear that it will restrict access to their own spectrum. Also, transaction costs associated with sub-letting a license are substantial. Legal and filing fees alone run thousands of dollars. In addition, the rules regarding service and emission masks are daunting to novice users.

Idle spectrum is one consequence of the current policy for spectrum management. A second is that it erects formidable entry barriers to the market for wireless services. This is due in part to the high degree of complementarity among spectrum licenses. To offer a wireless communication service over a broad coverage area, a potential entrant must acquire a package of associated spectrum licenses. Assuming the service would use mobile devices operating within a given band, the entrant must then bid for the same spectrum across different geographic regions. Therefore, the value a provider obtains from a license is contingent on the bundle of licenses already owned. The resulting high cost of spectrum combined with the

\footnotetext{
${ }^{4}$ These rules have subsequently been refined in [18], [19].
} 
high infrastructure investment makes it difficult to enter the market on a small scale (e.g., within a small geographic region). Hence the current cellular market is confined to service providers that can make an initial investment of several billions of USD. The limited amount of competition means that service providers can exert considerable influence over third-party hardware and software providers wishing to introduce new services (the "walled garden" concept). ${ }^{5}$

The underutilization of spectrum coupled with developments in spectrum-agile mobile devices has prompted a number of proposals for managing spectrum. On one side are proposals based on exclusive use [4], [14], [15], [23] and secondary markets [3], [8], [31], [36], [42]. Both presume some notion of spectrum property rights. On the other side are various proposals based on the commons model [6], [13], [26], [32], [33], [43] in which any user can access spectrum provided that they obey basic "spectrum etiquette" rules, such as a power mask. ${ }^{6}$

In this paper we reconsider the spectrum allocation problem without existing regulatory constraints. ${ }^{7}$ We start by providing general motivations for introducing spectrum markets. Namely, a basic policy choice is whether to define and enforce spectrum property rights, which can be traded. From a social welfare point of view, this choice ultimately depends on whether or not spectrum is scarce, i.e., if demand for it exceeds supply when it is free. If spectrum is abundant, then it can be made freely available (subject to appropriate power constraints), as in the commons model. If spectrum is scarce, then an allocation mechanism, which coordinates allocations and usage becomes desirable. $^{8}$

Whether a particular band of spectrum is scarce depends on propagation characteristics and the transmitted power, which determines range and interference levels, and the nature of the traffic demands. At high enough frequencies (say, above $3 \mathrm{GHz}$ ) the range is sufficiently small that interference is not a major concern unless the density of wireless devices becomes very large. Therefore the commons model is likely to be appropriate for those frequencies. On the other hand, low frequencies have longer range, compromising frequency reuse, so that the commons model is probably inappropriate for commercial wireless services (e.g., see [9], [40]). For those frequencies, an infrastructure of Access Points (APs) is needed to coordinate transmitters and control interference.

To determine whether spectrum should be viewed as a scarce resource, we estimate in Section III the achievable rate per user assuming that spectrum currently assigned to nongovernment services between $150 \mathrm{MHz}$ and $3 \mathrm{GHz}$ is available for commercial services. The calculation assumes a fixed

\footnotetext{
${ }^{5}$ Even without spectrum policy reform, emerging cognitive radio technology has the potential to alleviate this problem, since a service provider would no longer need to use the same band over its coverage region.

${ }^{6}$ As several papers have pointed out, hybrid models that bridge these two extremes are also possible [35].

${ }^{7}$ Moreover, we do not address the formidable problem of transitioning from the current regime to a new regime (e.g., see [10]).

${ }^{8}$ Of course, as argued in [16], in addition to scarcity, the transaction costs for implementing a given policy are also important in making this choice.
}

density of access points, and accounts for interference between adjacent cells using standard propagation models. Although the answer depends on assumptions concerning frequency reuse in different parts of the band, the power constraint, and the distance of the user from the cell boundary, we conclude that extensive spectrum sharing in the range considered (with a managed infrastructure) could provide a few Mbits/sec per user. While this is a relatively large number for many types of services, including medium-grade video, it is small enough that some distributed spectrum management is likely to be necessary to avoid a tragedy of the commons.

Spectrum markets are subsequently described, along with implications on markets for wireless services. Specifically, we discuss a two-tier market for spectrum assets: in the top tier spectrum property rights at particular locations (APs) are traded among spectrum owners (as in a commodities market); in the bottom tier spectrum is rented or leased to service providers at particular APs via a spot market run by spectrum brokers. The relationships among spectrum providers, equipment providers, and wireless service providers are discussed, along with issues that may arise. We conclude that separating the market for spectrum from the market for wireless services would allow efficient and flexible allocation of spectrum, while lowering entry barriers for providing new wireless services.

\section{The Motivation for Spectrum Markets}

From an economic perspective a common objective of any resource allocation is to maximize efficiency, meaning the total utility derived from the allocation (e.g., summed over all agents requesting the resource). Essentially, an efficient allocation assigns resources to those agents who value it the most. Determining an efficient spectrum allocation is complicated by a number of factors. Those are mainly a consequence of propagation characteristics, which can vary substantially across frequency and locations, and variations in application requirements (e.g., voice, internet, broadcast, emergency, etc). Hence the portion of the spectrum most suitable for a particular application can change over location and time. Furthermore, the relative value of the applications to wireless customers can also change across location, times, and user groups. Moreover, the mapping of application requirements to spectrum depends on the available technology and its costs, which changes over time.

Allocations of spectrum to different applications are typically made by government agencies, such as the FCC in the U.S., and are static, i.e., they typically apply for many years. That has led to inefficient use of the spectrum resource for the following reasons:

1) For a given service (e.g., defense, emergency, cellular, etc) it is difficult to predict actual usage over the duration of a spectrum license (i.e., multiple years), which makes it difficult to match spectrum assignments to associated demand.

2) Static assignments cannot exploit statistical multiplexing of traffic across different applications over shorter time scales. Namely, even if the spectrum assignments are 
able to match average long-term demand, there are typically large fluctuations in demand, which lead to inefficient allocations over shorter time periods.

3) Each spectrum asset is currently assigned to the same agency or application, independent of location, whereas demand for a particular application typically varies substantially across different locations (e.g., urban vs rural).

4) It is difficult for a centralized authority to obtain an accurate estimate of the economic utility associated with a particular static assignment.

Many, if not all, of these problems with centralized allocation are not unique to spectrum. (For example, some also apply to land assets.) It is well-recognized that these types of inefficiencies can be alleviated through the use of market mechanisms. Spectrum markets have been previously discussed in [2], [10], [11], [14]-[18], [29], [36], [41], [44]. In [3], [31], [36] secondary markets for spectrum are considered in which a primary user (licensee) is able to rent or lease spectrum on a short-term basis to secondary users. In those scenarios the secondary market is managed by the primary user (spectrum licensee), who may be able to increase revenue by renting assigned,but unused spectrum.

Here we consider the scenario in which spectrum is made available for sharing among many different applications across a large geographic region. We do not assume any constraints on how the spectrum can be assigned or managed. Our only assumption is that the spectrum is partitioned into a set of spectrum assets that can be allocated among agents (service providers) at different locations. Specifically, the spectrum market could be associated with a network of Access Points (APs), which may include the current cellular infrastructure of base station towers. Each AP would have a set of particular spectrum assets, which are allocated among agents by a spectrum broker. Our main focus is on the scenario where spectrum is used to provide network access to users via these APs. Such connections could provide the commercial wireless services available today including voice, data, and broadcast radio or television. ${ }^{9}$

\section{A. Spectrum Markets versus The Commons Model}

As previously mentioned, the spectrum markets discussed are intended for frequencies appropriate for micro- to macrocellular types of systems. At higher frequencies (e.g., above $3 \mathrm{GHz}$ ), where the propagation range over a very large bandwidth is confined to a few meters with an appropriate power mask, interference management is not a major concern, so that the commons model is appropriate. Of course, the commons model could be used at all frequencies, eliminating the need for spectrum markets altogether (e.g., see [6], [26]). There are two drawbacks with this proposal.

First, the propagation range associated with signals at lower frequencies is more difficult to predict than for frequencies where the commons model is currently used (i.e., the $2.4 \mathrm{GHz}$

\footnotetext{
${ }^{9}$ This access need not be "one-hop", and may allow for various forms of multi-hop connectivity.
}

and $5 \mathrm{GHz}$ bands). That is, depending on the environment, such signals may propagate much farther in certain directions than in other directions. This makes interference management difficult because in a commons model, there are no restrictions on where APs can be deployed. This drawback could be eliminated by combining the commons approach at lower frequencies with a cellular infrastructure, which restricts AP locations, or restricts the use of particular frequencies at certain locations. Subject to those constraints, spectrum could otherwise be freely available.

The second drawback is that as the demand for wireless services grows, demand may eventually exceed supply, creating excessive interference and lowering overall utility. This drawback is the primary motivation for the creation of spectrum markets. The apparent scarcity of spectrum at present, however, is not by itself evidence that a commons approach may be inadequate. This scarcity may be an artifact of the current inefficiencies created through centralized spectrum allocations [26]. We explore this issue in the next section by estimating the achievable rate per user that would be available if extensive sharing of lower frequencies suitable for micro/macro cellular services were allowed.

Although the rate calculation in the next section gives a rough indication of whether spectrum is scarce or abundant, ultimately the value of a particular spectrum asset can be determined only through a market. If spectrum is truly abundant, then the prices of all spectrum assets will fall to zero, in which case the spectrum market reduces to the commons model [16]. Of course, in general prices of spectrum assets should vary across frequencies and locations according to variations in demand and interference levels.

The preceding discussion implies that the frequencies at which the spectrum market transitions to a commons model can be automatically determined by the spectrum market (e.g., see also [9]). Namely, at high enough frequencies the price of the spectrum assets should be zero, since the propagation range is highly confined, and therefore useful to a small number of devices.

\section{B. Spectrum versus Technology Costs}

The efficiency (or utility) of a spectrum allocation depends not only on the allocation itself, but also on the costs of devices and systems which use the spectrum. This is reflected in the design of current wireless equipment and standards, which have been developed under the assumption that spectrum scarcity poses a major limitation on system capacity and revenues. The high cost of spectrum has led to the development of cellular wireless standards with sophisticated modulation, coding, and multiple access schemes, which attempt to maximize spectral efficiency (rate per unit bandwidth).

Inexpensive spectrum, obtained through a spectrum market, would likely motivate the design of inexpensive wireless technologies, which operate at much lower spectral efficiencies, compared with current cellular standards. Furthermore, there is a fundamental tradeoff between bandwidth efficiency and power efficiency. This means that given a target information 
rate, additional bandwidth enables a reduction in required transmit power, which in turn reduces interference.

Hence we conclude that the cost of spectrum, as determined by a spectrum market, would influence the cost of device design and standards development. The reverse should also be true, i.e., the cost of equipment and system design should influence the cost of spectrum. Namely, increased spectrum sharing should lower the cost of spectrum, but that will be offset by the increase in demand due to the deployment of less expensive wireless systems that require more bandwidth. Hence the cost of equipment development should be matched in some sense to the cost of spectrum. The net result of this interaction will be to lower the cost of providing wireless services to the consumer.

\section{IS SPECTRUM SCARCE?}

A key factor that should determine appropriate spectrum policy is whether spectrum is indeed a scarce resource [16]. The answer to this depends in part on regulatory and economic considerations, but is ultimately a technical problem of determining if network architectures can scale effectively as their applications grow [38]. Here, we address this question for a specific network architecture, namely communication from an infrastructure of base stations or access points (APs), which represents the primary network architecture deployed today for commercial wireless services. ${ }^{10}$

To determine whether or not spectrum is a scarce resource, we give a rough estimate of what rates could be provided with more extensive spectrum sharing. Namely, we assume that all spectrum between $150 \mathrm{MHz}$ and $3 \mathrm{GHz}$ is pooled for commercial services, excluding spectrum currently assigned for military and government use. The particular bands used in the calculation are shown in Table I. Note that broadcast television bands are included in this list. Demand for those services might be satisfied by a combination of wire-line cable connections combined with the wireless infrastructure assumed here.

We also assume that the APs are deployed with a particular frequency reuse patterns for interference mitigation. This should give an optimistic indication of what rates are achievable with full coordination among service providers; a relatively low rate per user indicates that spectrum is scarce, and needs to be carefully managed, whereas a very high rate indicates that relatively simple spectrum management schemes (e.g., the commons model) are likely to be adequate.

\section{A. Cellular Model}

To compute an achievable rate per user, a cellular topology with hexagonal cells (See Figure 1) is assumed over which both APs and users are uniformly spread with densities $\rho_{a p}$ and $\rho_{u}$, respectively. Each AP serves the same number of users. Here we focus on the achievable rate for the downlink, i.e. communication from the AP to each user. We expect similar results for the uplink.

\footnotetext{
${ }^{10}$ Moreover, this architecture has better scaling properties than alternatives, such as mesh networks, provided that sufficient access points are used.
}

We make the following assumptions:

1) The entire set of available frequencies in Table $I$ is quantized into $1 \mathrm{MHz}$ pieces, which are allocated across the APs according to a standard frequency reuse pattern.

2) Each AP transmits with uniform power spectral density over the set of assigned channels.

3) Each AP applies Time-Division Multiple Access (TDMA) to multiplex users within the cell. Hence with $n$ users per cell, the AP transmits to each user $1 / n$ of the time.

4) Interference from only neighboring cells is taken into account. Also, we assume that the signal attenuation is determined according to large-scale propagation models, and do not account for random fluctuations (fading).

For a particular $1 \mathrm{MHz}$ channel at frequency $f$, the rate per $1 \mathrm{MHz}$ bandwidth for a particular user at distance $d$ from the AP is assumed to be the Shannon rate

$$
R(d, f)=\frac{1}{n} \log (1+\operatorname{SINR}(d, f))
$$

where $n=\rho_{u} / \rho_{a p}$ is the number of users per cell. Here, $\operatorname{SINR}(d, f)$ is the Signal-to-Interference-Plus-Noise Ratio given by

$$
\operatorname{SINR}(d, f)=\frac{P_{r}(d, f)}{N_{0}+\sum_{i \in \mathcal{I}} P_{r}\left(d_{i}, f\right)},
$$

where $P_{r}(d, f)$ is the received power density for a user at distance $d$ at frequency $f, \mathcal{I}$ is the set of interfering APs, and $P_{r}\left(d_{i}, f\right)$ is the received interference power density from the $i$-th interfering AP. $N_{0}$ is the power spectral density of the additive noise.

This rate assumes optimal coding and delay-tolerant applications and so gives an upper-bound on the rate that can be obtained. ${ }^{11}$ In principle, we can account for channel variations (fading), practical coding schemes with delay constraints, interference from more distant cells, etc. by adding an appropriate margin to the SINR.

The achievable rate for a particular user depends upon where they are in the cell. The lowest rate corresponds to a user at the corner of a cell, as illustrated in Figure 1. From the hexagonal geometry we have

$$
\begin{aligned}
& \operatorname{SINR}(r, f)= \\
& \quad \frac{P_{r}(r, f)}{N_{0}+2\left\{P_{r}(r, f)+P_{r}(2 r, f)+P_{r}(\sqrt{7} r, f)\right\}},
\end{aligned}
$$

where $r$ is the cell radius. ${ }^{12}$

To determine $P_{r}(r, f)$ we use Hata's outdoor propagation model for the frequency range $150 \mathrm{MHz}$ to $1.5 \mathrm{GHz}$, and its extension to PCS for $f>1.5 \mathrm{GHz}$ (see [37, Ch. 4]). The rate is then obtained by quantizing the spectrum bands shown in

\footnotetext{
${ }^{11}$ There are techniques which could enable users to achieve higher rates than this, such as the use of multiple antennas (beamforming) combined with cooperative techniques for avoiding interference. The gain from such approaches may be best realized when neighboring APs are managed by a single entity.

${ }^{12}$ For hexagonal cells the density of access points is $\rho_{a p}=1 /\left(2.6 r^{2}\right)$.
} 
TABLE I

FREQUENCIES USED TO CALCULATE ACHIEVABLE RATES.

\begin{tabular}{l|l|}
\hline Broadcasting TV (total: $348 \mathrm{MHz}$ ) & $174-216 \mathrm{MHz}, 470-608 \mathrm{MHz}, 614-764 \mathrm{MHz}, 776-794 \mathrm{MHz}$ \\
\hline Fixed, Mobile, Satellite, Amateur & $150.8-157.0375 \mathrm{MHz}, 157.1875-162.0125 \mathrm{MHz}, 173.2-173.4 \mathrm{MHz}, 450-460 \mathrm{MHz}$, \\
(total: 669.7625 MHz) & $764-776 \mathrm{MHz}, 794-902 \mathrm{MHz}, 928-932 \mathrm{MHz}, 935-941 \mathrm{MHz}, 944-960 \mathrm{MHz}$, \\
& $1390-1395 \mathrm{MHz}, 1427-1429 \mathrm{MHz}, 1850-2025 \mathrm{MHz}, 2110-2200 \mathrm{MHz}$, \\
& $2300-2310 \mathrm{MHz}, 2385-2417 \mathrm{MHz}, 2450-2483.5 \mathrm{MHz}, 2500-2655 \mathrm{MHz}$ \\
\hline
\end{tabular}

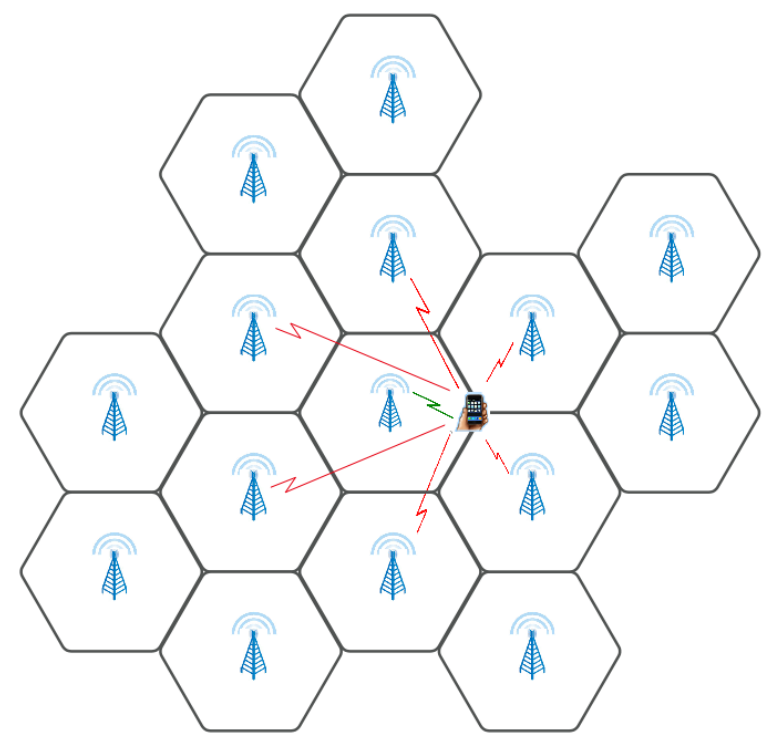

Fig. 1. Cellular system used to calculate achievable rates. The location of the worst-case user with the lowest rate is shown. Only interference from the neighboring cells is taken into account.

Table I into $1 \mathrm{MHz}$ pieces, and summing the rate function over those bands. To account for losses expected in practice (e.g., due to other channel impairments) a $6 \mathrm{~dB}$ margin is subtracted from the SINR. Also, we assume a fixed power per unit area, i.e., the total power across the area covered does not scale with the density of APs. That also constrains the background interference level.

\section{B. Achievable Rate Results}

Results from the preceding calculation are shown in Fig. 2. For these plots the transmit power density $P_{t}=-40 \mathrm{dBm} / \mathrm{Hz}$ for all APs, the noise power spectral density $N_{0}=-174$ $\mathrm{dBm} / \mathrm{Hz}$, the base station antenna height is $30 \mathrm{~m}$, and the receiver antenna height is $1 \mathrm{~m}$.

Figure 2(a) shows achievable rates for the worst-case user at a corner point of the cell versus user density. Different curves are shown for different values of the cell radius $r$. For these plots the frequency reuse factor $N$ is chosen such that the rate at each frequency is maximized. ${ }^{13}$ Hence the rate per user decreases as the cell radius increases. (This is not necessarily true if $N$ is fixed, since the interference decreases with the cell

\footnotetext{
${ }^{13} N$ is chosen from $1,3,4$ or 7 based on the cellular assumptions.
}

radius.) As a specific example, for a large city like Chicago, which has a population density of 4250 people per square $\mathrm{km}$ [7], the worst-case rate per user increases from 0.3 to 1.8 Mbps as the cell radius shrinks from $500 \mathrm{~m}$ to $200 \mathrm{~m} .{ }^{14}$

Figure 2(b) shows how the achievable rate varies with the distance from the AP. Namely, the achievable rate per cell at different distances from the AP is shown versus the cell radius. (The rate per user is then obtained by dividing this rate by the user density.) These results indicate that the rate increases by about $50 \%$ in moving from the edge of the cell to distance $r / 2$, and more than doubles if the distance decreases to $r / 4$.

\section{Discussion}

The preceding results indicate that if a cellular infrastructure with cell radii less than $200 \mathrm{~m}$ has access to all of the bandwidth in Table I, then rates well above 1 Mbps could, in principle, be made continuously available to every member of a dense urban population. Furthermore, the achievable rate increases substantially with the density of APs, and with the fraction of inactive users (as opposed to assuming all users are active).

From an applications perspective, the range of rates indicated here are sufficient to support a wide range of near-term mobile services. One might therefore conclude that simple allocation schemes, such as those based on a commons model, may be adequate for spectrum allocation. However, spectrum markets are likely to be needed for the following reasons:

1) The rates shown in the preceding section are optimistic in that the availability of a managed infrastructure with coordinated frequency reuse has been assumed. Also, we have not directly accounted for channel impairments associated with large-scale fading and mobility.

2) We have assumed an extreme case in which a large amount of spectrum currently assigned to many different applications is pooled for shared use. If less spectrum is available, then the rates decrease accordingly. For example, given sufficient demand for broadcast services, it may be desirable to set aside spectrum for that

\footnotetext{
${ }^{14}$ In this case, the spectrum efficiency is about $0.7 \mathrm{bps} / \mathrm{Hz}$ per cell. This is small compared with the spectrum efficiency of mobile WiMax (IEEE 802.16e), which is about $1.88 \mathrm{bps} / \mathrm{Hz}$ [20] in the best scenario. This is due to several reasons: 1) We assume simple spectrum spread modulation; 2) Here the spectrum efficiency corresponds to the worst case in which a user is located on cell boundary; 3) We assume omni-directional antennas without cell sectorization; 4) The spectrum efficiency of WIMAX does not account for inter-cell interference.
} 


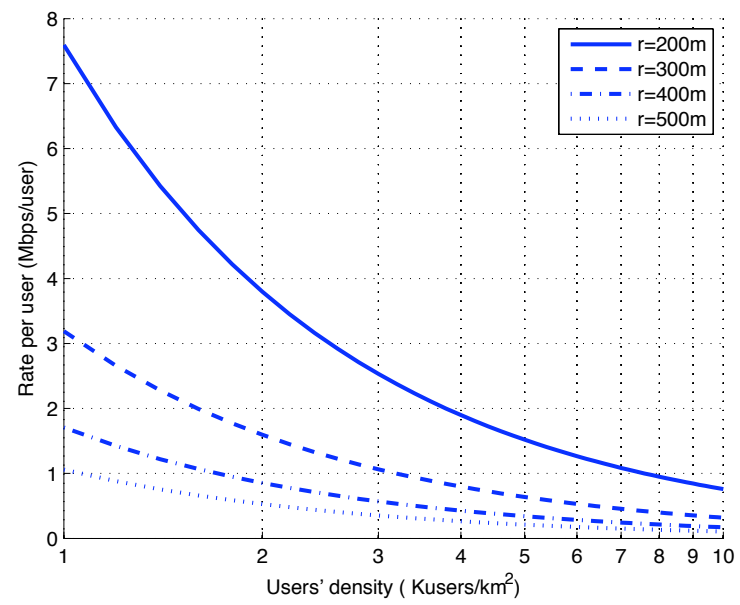

(a) rate/user vs $\rho_{u}$

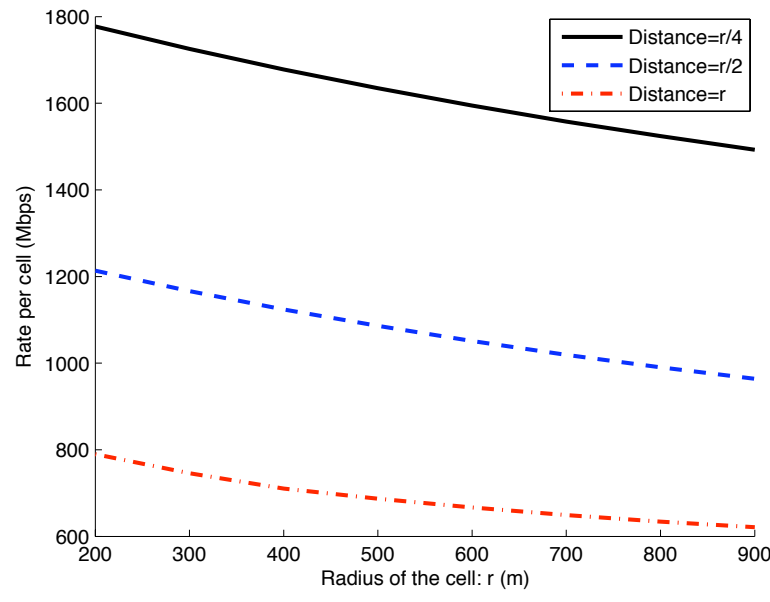

(b) rate/cell vs radius

Fig. 2. Achievable rates with a cellular infrastructure assuming all frequencies shown in Table I are available for sharing: (a) worst-case rate per user versus user density; (b) rate per cell versus cell radius.

purpose. This would reduce the total rate available for non-broadcast services to each user. ${ }^{15}$

3) As discussed in Section II-B, additional spectrum can be exploited to simplify system design, e.g., by using modulation and coding schemes that operate at rates well below the Shannon rate. Additional bandwidth also enables a reduction in transmit power and associated interference.

4) Although the rates reported here may seem large (especially when divided among a relatively small set of active users), it is possible that in the long-term new applications may arise that require rates on the order of (or beyond) what are indicated here.

Hence, we conclude that even with extensive spectrum sharing and with coordination of spectrum resources across APs, the demand for spectrum may exceed the supply as users, applications, and systems proliferate. We therefore discuss how spectrum markets might be defined that achieve efficient allocations and benefit consumers of wireless services.

\section{Spectrum Assets: Property Rights}

Spectrum sharing through a market mechanism presumes an appropriate definition of spectrum property rights. This notion was originally proposed by Coase [12], and subsequently modified by others [21], [22], [30]. The purpose of property rights in spectrum is to limit the amount of interference an owner (or licensee) receives from transmitters associated with other owners (or licensees).

\footnotetext{
${ }^{15}$ This would in fact increase the total rate that a user can receive since broadcast services require a single transmission to reach all users in a cell. Furthermore, many current broadcast services (e.g. television) could be offered as multicast services over this type of cellular architecture. This would make more efficient use of spectrum since the service would only need to be transmitted in a given cell provided that someone in the cell wanted to receive
}

From the point of view of economic efficiency, the definition of a spectrum asset should satisfy the following criteria.

1) Property rights associated with the ownership of spectrum assets should be clearly defined and easily enforced.

2) An agent allocated spectrum should be able to determine in a straightforward way the associated benefit.

3) The definition should facilitate efficient allocations (e.g., avoid "natural" monopolies).

4) The cost of equipment to exploit the asset should be as low as possible so as to enable entry.

The difficulty with defining property rights that satisfy the first criterion is that radio signals are not easily confined with respect to location and frequency. While the strength of a signal declines with distance, it is not guaranteed to expire at a preset threshold. ${ }^{16}$ Hence, between one defined geographic area and another there will be intermittent interference. A second source of interference is from transmitters occupying adjacent bands in the same geographic area. To some extent this depends on the ability of the receiver to attenuate outof-band signals, although this type of interference is mostly determined by the power mask for the adjacent spectrum asset. Of course, this adjacent-channel interference can sometimes extend beyond the adjacent channels.

The difficulty with satisfying the second criterion is that the utility associated with a spectrum asset for a particular transmission depends on the application, the propagation environment (distance from the receiver, multipath) as well as interference, which is a function of how the spectrum asset is allocated among other agents. Hence in practice these criteria must be compromised when defining property rights that form the basis of an appropriate market.

\footnotetext{
${ }^{16}$ In particular, the higher the frequency, the more rapidly the signal strength declines.
} 


\section{A. Power Mask}

The forms of interference just described can be managed by limiting the power used to transmit signals. Indeed, some type of limit on the radiated power (both within the assigned band as well as outside the band) is the basis for interference management in most current wireless systems today. We propose using a similar definition here, i.e., a spectrum asset is assumed to be defined in terms of a power mask over a set of frequencies for a given time duration within a given geographic area. This type of definition can serve as the basis for the spectrum markets described in the next section with the following interpretations:

1) The power mask for an AP may depend on factors such as the antenna height and the distance of the AP to the boundary of the given geographic area. For example, the power limit could decrease for a transmitter near the boundary since such a transmitter would generate greater interference for an AP in an adjacent area, which is assigned an overlapping spectrum asset.

2) The power mask may also vary with frequency and time. Namely, because attenuation increases with frequency, the power limit may increase as well. Furthermore, the power limit may increase when the system is lightly loaded, i.e., at those times the spectrum owner can transmit with higher power. Similarly, we expect that the power will decrease as the user density increases (mainly due to an increase in the density of APs). ${ }^{17}$

Defining spectrum property rights in this way does not give adjacent users a hard guarantee about the received interference level. Indeed, as discussed in prior work (e.g., [30]), random variations in propagation characteristics and usage patterns over different locations and frequencies would make such a guarantee difficult, if not impossible to enforce. It does however give a user a reasonable expectation about the interference to be expected. Moreover, if a provider requires a stronger guarantee about the interference, it would have the option of simply acquiring the neighboring spectrum assets itself so as to ensure that no other transmitter is using them. ${ }^{18}$

\section{B. Owning versus Leasing}

For the spectrum markets to be described there is an important distinction between owning and leasing or renting a spectrum asset. The definition of the spectrum asset depends on this distinction. Namely, ownership of a spectrum asset implies a long duration (e.g., many years). However, spectrum assets can be rented or leased by the spectrum owner. The time duration of the spectrum asset being rented can vary across frequencies, agents, and locations, and determines

\footnotetext{
${ }^{17}$ Here we have focused on the power mask for an AP within a given area. A similar approach could be used for the uplink, i.e., each mobile user could be constrained to satisfy a power mask, which again could vary with distance, frequency, and time.

${ }^{18} \mathrm{~A}$ similar argument can be applied to the problem of co-channel interference. A provider can have the option of either investing in better receivers to remove co-channel interference or using cheaper receivers and buying adjacent "guard-bands" to protect it from interference.
}

market dynamics. Namely, a short duration (say, less than a day) may be associated with a spot market for short-term commercial use (analogous to electricity markets [1], [25]), whereas a long duration (e.g., years) may be associated with broadcast services that require continual use of spectrum. Note, in particular, that a spectrum owner could conceivably decide to switch applications (e.g., migrate from broadcast to cellular), or sell spectrum rights to another owner once a rental agreement has expired.

Note that spectrum assets could also be aggregated or disaggregated. Namely, a spectrum owner (or renter) may wish to aggregate spectrum assets at adjacent locations in order to manage more effectively the associated interference. Conversely, a spectrum owner may disaggregate spectrum by renting segments across different service providers and applications. It would then be up to the owner to define an appropriate power mask for each segment. This could be done in any way provided that the total usage still satisfies an overall power mask for limiting interference into neighboring locations associated with other owners.

Once property rights for spectrum are defined, these rights are best allocated via a market. Namely, a centralized scheme for allocating spectrum would have to collect and process an enormous amount of information about user preferences, propagation characteristics, potential applications, and different technologies, which far exceeds practical limitations. A market solves this problem of information exchange in a distributed way by allowing the agents themselves to determine the assets to exchange based on local knowledge about usage, expected interference, and applications.

\section{Market Structure}

Allowing spectrum property rights to be flexibly defined and traded would produce a sea change in the market structure for wireless services. The most visible of those changes would be the separation of spectrum ownership from the provision of wireless services. As previously discussed, it may improve efficiency if spectrum is not tied to particular services. Here we describe additional features of the wireless service market, which we expect would arise as a consequence of this change in policy. Namely, the market for wireless services would consist of at least three dependent segments: spectrum owners, service providers, and end customers. The interface between service providers and spectrum owners could be through a spectrum broker, which manages spectrum assets and determines local allocations among service providers.

1) Spectrum Owners: Spectrum assets at a particular AP would be traded according to a conventional market mechanism, as in a commodity market. An issue, which arises with a spectrum asset, is that its value depends on the locations of nearby APs, which can change over time. This may encourage aggregation of spectrum assets across neighboring locations, as discussed in the previous section. Strong interference between nearby APs with different owners may have to be resolved through additional negotiations. We return to this issue in Section VI. 
Spectrum owners would be motivated to rent their assets to applications that generate the most revenue. By allowing spectrum owners to trade spectrum assets, each asset could be reassigned to applications that generate higher revenue, or alternatively, to groups that want to purchase spectrum for non-commercial purposes (e.g., community broadcast). Furthermore, many spectrum assets would likely be available at each AP and the entry and exit cost for trading spectrum would presumably be low, creating a competitive market. Hence spectrum usage would be determined by market supply and demand, and the price of a particular spectrum asset would be tied to the expected revenue it could generate.

2) Service Providers: A service provider offers a set of wireless services to end customers through a particular pricing scheme. With independent spectrum markets a service provider could purchase (rent) spectrum on a short-term basis. It would then need to notify the end-user device what particular band to use. ${ }^{19}$ As a consequence, a service provider need not build out a national footprint of APs, which use the same spectrum. The spectrum could be rented via the spectrum spot market at desired locations.

We remark that the service provider could also choose to rent the necessary equipment at an AP from an equipment manufacturer. (That cost would also account for the cost of the tower on which it is mounted.) Hence the combination of spectrum markets and equipment rentals could dramatically lower the entry (sunk) costs for a service provider. That would potentially increase competition along with service options. ${ }^{20}$

Service providers may also provide an arbitrage function for customers. Namely, prices for spectrum rental may experience large short-term fluctuations, whereas end customers typically prefer predictable (e.g., flat-rate) pricing plans. Hence the pricing plans offered by the service provider would account for those fluctuations by including a premium, which guards against the possibility of high short-term prices. (Of course, for delay-insensitive traffic, the service provider could wait to see if short-term prices fall.) A service provider may also choose to negotiate longer-term contracts for spectrum with the spectrum owner to provide more reliable Quality of Service.

We also observe that the arbitrage function may be performed by independent agents who purchase spectrum on the spot market and resell to spectrum providers. That would shift the burden of forecasting and speculating on spectrum prices across time and locations away from the service providers, and may help to stabilize prices for the service providers. In addition, the arbitrage agents may be held responsible for compensating service providers during periods when spectrum is unavailable due to excessive demand.

In this way, spectrum assets are treated as commodities, which can be used for a variety of applications over differ-

\footnotetext{
${ }^{19}$ Of course, this requires some signaling overhead, but could likely be done with minimal cost.

${ }^{20}$ Furthermore, the AP provider may lease equipment from the manufacturers. The allocation of available RF equipment at an AP to service requests might then be done through an "equipment broker". Conceivably this function could also be combined with that of the spectrum broker.
}

ent locations and times. Ideally, this flexibility would allow available spectrum to be matched to demand for applications.

3) Role of Spectrum Broker: The spot market for spectrum assets at each AP would be managed by a spectrum broker. The spectrum broker therefore determines how spectrum assets are allocated among service providers, and how much each agent pays for a spectrum asset. The amount a particular agent pays may be determined either through negotiations (i.e., an auction mechanism) or through announced prices. In the former scenario, the broker can serve as the auctioneer, which collects bids, and announces the allocation. In the latter (pricing) scenario the broker can adjust prices for the spectrum assets to clear the market. In either scenario, the protocol for information exchange (bids for spectrum assets or price announcements) could be automated and run on a spectrum server (e.g., see also [5], [24], [27], [28], in which the concepts of spectrum broker and server are also used in a related context).

\section{ISSUES}

Here we briefly discuss issues, which are likely to arise in connection with the market structure for spectrum previously described. This discussion is not intended to be complete. Namely, there are other important issues, which we do not address here (e.g., the transition to such a spectrum market), and there are likely to be other unforeseen issues that arise.

\section{A. Interference Management}

As mentioned in Section V, some form of interference management is needed among nearby APs for which the same spectrum is owned by different entities (adjacent owners). At a basic level this is accomplished by appropriately defining the spectrum power mask. However, this may be inadequate depending on the density of APs, propagation characteristics, and demand for spectrum at those locations. The solution in severe cases (e.g., dense urban locations) may entail renegotiating and/or aggregating spectrum property assets at those locations, or cooperation among adjacent owners. For example, adjacent owners may negotiate cross-rental agreements for the same spectrum, or alternatively, agree to pay "interference charges" to each other, which reflect the externality they are causing to neighboring owners. (Such interference charges are in fact necessary to achieve economic efficiency.)

Adding an AP at a particular location would also have to be coordinated with existing nearby APs. It may be necessary for spectrum owners at the new location to negotiate power masks with the existing owners. ${ }^{21}$ We remark that similar situations arise today at boundaries of regions operated by different wireless service providers. Contentious situations arise infrequently, since there is substantial mutual benefit associated with local cooperation. The likelihood of those contentious situations may increase with an increase in number

\footnotetext{
${ }^{21}$ This issue is analogous to the obstruction of a condominium view due to new construction. A difference is that a spectrum asset is more flexible, which may ease negotiations.
} 
of service providers, but would decrease as the amount of spectrum available for sharing increases.

\section{B. Local Coordination}

As wireless services providers and APs proliferate, functions requiring local coordination, such as handoff, may require modifications of the techniques currently used in cellular systems. This is due to the possibility of handing off to one of several service providers, which may have access to different parts of the spectrum at different times. Hence providing extended coverage to mobile users would require cooperation with neighboring service providers. Of course, this type of cooperation already exists for cellular systems, albeit on a smaller scale than what would probably be needed in the market scenario considered. One possible approach is to incorporate the handoff function in the spectrum broker, which would assign those requests high priority. Related Functions such as registration and paging could be handled similarly.

Local coordination issues would also arise with billing, although it seems likely that current methods used for billing across different service providers (both wired and wireless) could also be used in such a distributed wireless services market.

\section{Regulatory Considerations}

Because spectrum markets should lower entry barriers to the wireless services market, thereby increasing competition, introducing such markets should ease the burden on regulators of wireless service providers. Still, there are conceivable scenarios in which coalitions of spectrum owners and equipment providers could act to restrict entry into the market. Namely, the owner of a tower for an AP could form a coalition with a spectrum owner and service provider, and restrict other service providers from renting spectrum at that location (or several locations). Some protection against such scenarios may be obtained by enforcing a segmentation of the markets for spectrum, equipment, and wireless services. ${ }^{22}$ However, given a large amount of spectrum to share at each AP along with other competing (e.g., wired) technologies for network access, forming such a coalition to restrict access in this way would likely be difficult.

Of course, existing laws aimed at anti-competitive behavior would also apply to the spectrum and wireless services markets. The interpretation of those laws in this new context would evolve along with evolving spectrum markets and associated wireless applications.

\section{CONCLUSIONS}

Allowing spectrum to be traded and rented at different locations would lead to major changes in markets for wireless services. A key consequence is that spectrum ownership could be separated from the provision of wireless services. That would facilitate the introduction of more diverse sets of service offerings, due to lower entry barriers, and may motivate the

\footnotetext{
${ }^{22}$ This is in contrast to current policy in which a service provider must own the associated spectrum.
}

design of different types of radio systems, which are less sensitive to constraints on spectral occupancy. Furthermore, markets for spectrum assets would allow the same spectrum to be allocated to different applications across locations and times, according to demand.

Here we have focused on the consequences of policies, which would allow spectrum markets. There are, of course, numerous issues that arise when considering how the transition to those policies may occur. Additionally, there are a number of associated regulatory issues that should be addressed in more detail. Such issues may be considered in future work.

\section{REFERENCES}

[1] "www.wesm.ph," Philippine Wholesale Electricity Spot Market.

[2] "Google intends to bid in spectrum auction if fcc adopts consumer choice and competition requirements," Letter to FCC Chair K. J. Martin, July 2007.

[3] A. Al Daoud, M. Alanyali, and D. Starobinski, "Secondary pricing of spectrum in cellular cdma networks," New Frontiers in Dynamic Spectrum Access Networks, 2007. DySPAN 2007. 2nd IEEE International Symposium on, pp. 535-542, 17-20 April 2007.

[4] P. Argyroudis, T. Forde, L. Doyle, and D. O'Mahony, "A policydriven trading framework for market-based spectrum assignment," in Proceedings of the Eighth IEEE International Workshop on Policies for Distributed Systems and Networks, 2007.

[5] J. Bae, R. Berry, and M. L. Honig, "Efficiency bounds for sequential resource allocation auctions," in Decision and Control, 2007. CDC '07 IEEE Conference on, 2007.

[6] Y. Benkler, "Overcoming agoraphobia: Building the commons of the digitally networked environment," Harvard Journal of Law \& Technology, vol. 11, no. 2, 1998.

[7] U. C. Bureau, "Summary file 1: 2000 census of population and housing," US Department of Commerce, Tech. Rep., 2000.

[8] M. Bykowsky, "A secondary market for the trading of spectrum: promoting market liquidity," Telecommunications Policy, vol. 27, no. 7, pp. 533-541, August 2003.

[9] M. Cave and W. Webb, "Spectrum licensing and spectrum commons: where to draw the line," in International Workshop on Wireless Communication Policies and Prospects: A Global Perspective, 2004.

[10] J. Chapin and W. Lehr, "The path to market success for dynamic spectrum access technology," Communications Magazine, IEEE, vol. 45, no. 5, pp. 96-103, 2007.

[11] _ _ "Time-limited leases in radio systems [topics in radio communications]," Communications Magazine, IEEE, vol. 45, no. 6, pp. 76-82, 2007.

[12] R. H. Coase, "The federal communications commission," Journal of Law and Economics, vol. 2, pp. 765-790, October 1959.

[13] M. Cooper, "The economics of collaborative production in the spectrum commons," New Frontiers in Dynamic Spectrum Access Networks, 2005. DySPAN 2005. 2005 First IEEE International Symposium on, pp. 379400, November 2005.

[14] A. S. de Vany, R. D. Eckert, C. J. Meyers, D. J. O'Hara, and R. C. Scott, "A property system for market allocation of the electromagnetic spectrum: A legal-economic-engineering study," Stanford Law Review, vol. 21, no. 6, pp. 1499-1561, June 1969.

[15] L. Doyle and T. Forde, "Towards a fluid spectrum market for exclusive usage rights," New Frontiers in Dynamic Spectrum Access Networks, 2007. DySPAN 2007. 2nd IEEE International Symposium on, pp. 620632, 2007.

[16] G. R. Faulhaber and D. Farber, "Spectrum management: Property rights, markets and the commons," in Telecommunications Policy Research Conference, 2003.

[17] FCC, "The development of secondary markets - report and order and further notice of proposed rule making," Federal Communications Commission Report 03-113, 2003.

[18] —, "Second report and order: Promoting efficient use of spectrum through elimination of barriers to the development of secondary markets," Federal Communications Commission Report 04-167, Sept. 2004. 
[19] — "Promoting efficient use of spectrum through elimination of barriers to the development of secondary markets - third report and order," Federal Communications Commission Report 07-52, 2007.

[20] D. Gray, "Mobile wimax: A performance and comparative summary," WiMax Forum, Tech. Rep., 2006.

[21] D. Hatfield and P. Weiser, "Property rights in spectrum: Taking the next step," 2005. [Online]. Available: http://ssrn.com/paper $=818624$

[22] D. N. Hatfield and P. Weiser, "Toward property rights in spectrum: The difficult policy choices ahead," 2006. [Online]. Available: http://ssrn.com/paper=975679

[23] T. W. Hazlett, "The wireless craze, the unlimited bandwidth myth, the spectrum auction faux pas, and the punchline to ronald coase's 'big joke': An essay on airwave allocation policy," Harvard Journal of Law \& Technology, vol. 14, no. 2, pp. 335-567, 2001.

[24] J. Huang, R. Berry, and M. L. Honig, "Auction-based spectrum sharing," ACM/Springer Mobile Networks and Applications Journal (MONET), vol. 11, pp. 405-418, 2006.

[25] S. Hunt and G. Shuttleworth, Competition and Choice in Electricity. John Wiley \& Sons, May 1996.

[26] N. Ikeda, "The spectrum as commons," November 2002, rIETI Discussion Paper No. 02-E-002.

[27] O. Ileri, O. Ileri, and N. Mandayam, "Dynamic spectrum access models: toward an engineering perspective in the spectrum debate," Communications Magazine, IEEE, vol. 46, no. 1, pp. 153-160, 2008.

[28] O. Ileri, D. Samardzija, T. Sizer, and N. Mandayam, "Demand responsive pricing and competitive spectrum allocation via a spectrum server," in First IEEE International Symposium on New Frontiers in Dynamic Spectrum Access Networks (DySPAN), 2005, pp. 194-202.

[29] E. R. Kwerel and J. R. Williams, "Moving toward a market for spectrum," Regulation: The Review of Business and Government, 1993.

[30] R. J. Matheson, "Principles of flexible-use spectrum rights," Journal of Communications and Networks, vol. 8, no. 2, pp. 144-150, June 2006.

[31] D. Niyato and E. Hossain, "Competitive pricing for spectrum sharing in cognitive radio networks: Dynamic game, inefficiency of nash equilibrium, and collusion," Selected Areas in Communications, IEEE Journal on, vol. 26, no. 1, pp. 192-202, Jan. 2008.

[32] E. Noam, "Spectrum auctions: Yesterday's heresy, today's orthodoxy, tomorrow's anachronism. taking the next step to open spectrum access," Journal of Law \& Economics, vol. 41, no. S2, pp. 765-790, October 1998.

[33] E. M. Noam, "The forth way for spectrum," Financial Times, May 29 2003.

[34] U. G. A. Office, "Strong support for extending fcc's auction authority exists, but little agreement on other options to improve efficient use of spectrum," Report to congessional Committees, GAO-06-236, December 2005.

[35] J. Peha, "Approaches to spectrum sharing," Communications Magazine, IEEE, vol. 43, no. 2, pp. 10-12, 2005.

[36] J. M. Peha and S. Panichpapiboon, "Real-time secondary markets for spectrum," Telecommunications Policy, vol. 28, pp. 603-618, 2004.

[37] T. S. Rappaport, Wireless Communications: Principles and Practice, 2nd ed. Prentice Hall, 1996.

[38] D. P. Reed, "How wireless networks scale: the illusion of spectrum scarcity," in International Symposium On Advanced Radio Technologies (ISART), 2002.

[39] I. Spectrum Bridge, "Spectrum licensing and secondary markets primer," White paper, available at www.spectrumbridge.com, March 2008.

[40] A. Tonmukayakul and M. B. H. Weiss, "A transaction cost analysis of secondary vs. unlicensed spectrum use," in Telecommunications Policy Research Conference, 2006.

[41] T. M. Valletti, "Spectrum trading," Telecommunications Policy, vol. 25, pp. 655-670, 2001.

[42] J. M. Ward, "Secondary markets in spectrum: Making spectrum policy as flexible as the spectrum market it must foster," CommLaw Conspectus: Journal of Communication Law and Policy, vol. 10, pp. 103-132, 2001.

[43] K. Werbach, "Open spectrum: The new wireless paradigm," new America Foundation, Spectrum Policy Program, Spectrum Series Working Paper \#6 October 2002.

[44] J. Williams, "A comparison of spectrum management by government vs market," Vehicular Technology Conference, 1981. 31st IEEE, vol. 31, pp. 305-310, April 1981. 